\title{
Intratumoral patterns of clonal evolution in gliomas
}

\author{
Ana Luísa Vital • Maria Dolores Tabernero • \\ Inês Crespo • Olinda Rebelo • Hermínio Tão • \\ Fernando Gomes • Maria Celeste Lopes • Alberto Orfao
}

Received: 23 April 2009/Accepted: 21 August 2009

(C) Springer-Verlag 2009

\begin{abstract}
Few studies have explored the patterns of clonal evolution in gliomas. Here, we investigate the cytogenetic patterns of intratumoral clonal evolution of gliomas and their impact on tumor histopathology and patient survival. Cytogenetic analysis of 90 gliomas was performed in individual tumor cells ( $>200$ cells/tumor) using multicolor ( $N=16$ probes) interphase-FISH. Overall, chromosome gains were more frequent than chromosome losses. Gains of chromosome 7 and/or EGFR amplification were detected in $91 \%$ of the cases, whereas $\operatorname{del}(9 \mathrm{p} 21)(77 \%)$ and $\operatorname{del}(10 \mathrm{q} 23)(78 \%)$ were the most frequent chromosome losses. Virtually, all cases (99\%) showed $\geq 2$ tumor cell clones, with higher numbers among high- versus low-grade gliomas $(p=0.001)$. Nine different cytogenetic patterns were found in the ancestral tumor clones.
\end{abstract}

Electronic supplementary material The online version of this article (doi:10.1007/s10048-009-0217-x) contains supplementary material, which is available to authorized users.

Maria Celeste Lopes and Alberto Orfao: both authors have equally contributed to this work and they should be both considered as last authors.

\footnotetext{
A. L. Vital $\cdot$ I. Crespo $\cdot$ M. C. Lopes

Centre for Neuroscience and Cell Biology,

University of Coimbra, Coimbra, Portugal

A. L. Vital • I. Crespo • M. C. Lopes

Faculty of Pharmacy, University of Coimbra,

Coimbra, Portugal

M. D. Tabernero $(\bowtie)$

Research Unit of the University Hospital of Salamanca,

Paseo San Vicente 58-182,

Salamanca, Spain

e-mail: taberner@usal.es

M. D. Tabernero $\cdot$ A. Orfao

Centre for Cancer Research (CIC IBMCC-CSIC/USAL)

and Department of Medicine, University of Salamanca,

Salamanca, Spain
}

In most gliomas, ancestral clones showed abnormalities of chromosome 7, 9p, and/or 10q and cytogenetic evolution consisted of acquisition of additional abnormalities followed by tetraploidization. Conversely, early tetraploidization was associated with low-grade astrocytomas-2/3 pilocytic and 3/ 6 grade II diffuse astrocytomas - and combined loss of 1p36/ 19 q13 with oligodendrogliomas, respectively; both aberrations were associated with a better patient outcome $(p=0.03)$. Overall, our results support the existence of different pathways of intratumoral evolution in gliomas

Keywords Glioma - Intratumoral heterogeneity . Clonal evolution $\cdot$ Cytogenetics

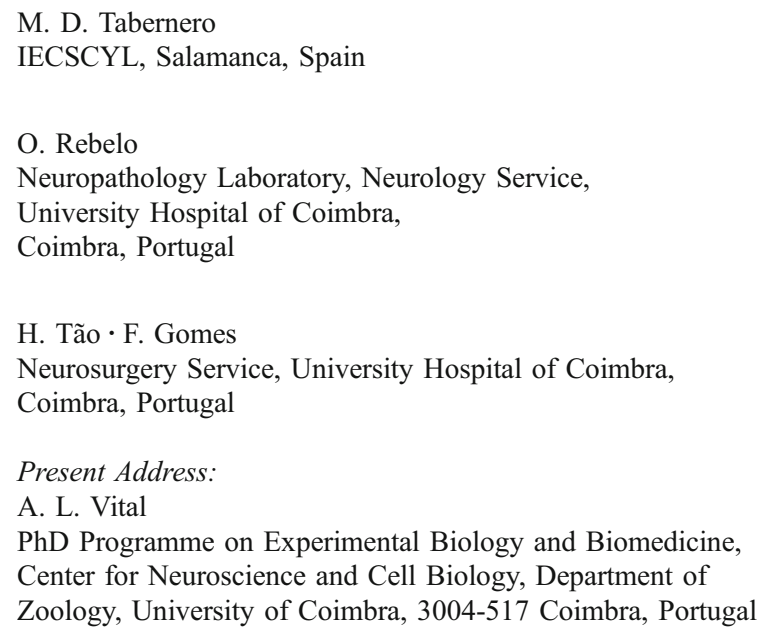




\section{Introduction}

Diffuse gliomas are the most common subtype of primary brain neoplasms in adults, accounting for around three quarters of all malignant, central nervous system tumors [1, 2]. Current classification of gliomas is based on morphological evidence of differentiation along the astrocytic $(\sim 70 \%$ of the cases) or less frequently, oligodendroglial, ependymal, or mixed cell lineages, and the histopathological degree of malignancy with $>80 \%$ of all diffuse gliomas being highgrade (grade III/IV) tumors [3, 4]. Despite significant advances in standard treatment modalities, the overall prognosis of patients with gliomas remains dismal $[5,6]$; however, a significant variation is observed in survival of individual patients [7-9], which cannot be explained just on the basis of tumor histopathology [10, 11]. In fact, in addition to tumor grade and the histopathological subtype, other factors such as patient's age and cytogenetics, are also recognized to be useful parameters in predicting patient outcome and survival [5, 9, 12-14].

At present it is well recognized that gliomas are genetically highly heterogeneous tumors, even within a single histopathological category $[15,16]$. Many studies have been reported so far, in which cytogenetic and molecular changes associated with human gliomas have been identified using different techniques [14, 17-21]. Among other alterations, the most frequently detected molecular abnormalities include gains of chromosome 7-with or without amplification of the epidermal growth factor receptor $(E G F R)$ gene- deletion and mutation of TP53, alteration of the retinoblastoma pathway involving the RB1 gene localized at chromosome 13q14, together with losses of the PTEN gene and hemizygous/homozygous deletion of the $p 16$ tumor suppressor gene at chromosomes 10q23 and 9p21, respectively [14, 22-26]. To date, two distinct, mutually exclusive molecular profiles have been identified, which would correspond to either de novo (primary) or secondary glioblastomas developing after progression of either a low-grade or an anaplastic astrocytoma [27, 28]. Accordingly, while primary glioblastomas show higher frequencies of p16 deletion, EGFR amplification and PTEN mutation with a relatively low frequency of TP53 abnormalities, secondary glioblastomas more frequently carry mutations of TP53 in association with abnormalities of the EGFR, p16, and PTEN genes [28, 29]. Despite this, no study has been reported so far in which the major pathways of acquisition of genetic abnormalities are systematically analyzed in glioma patients, at the intratumoral cell level $[8,30]$.

In the present study we have applied multicolor interphase fluorescence in situ hybridization (iFISH) for the analysis of multiple combinations of 16 different probes specific for DNA sequences of the most frequently altered chromosome regions, to gain insight into the intratumoral cytogenetic heterogeneity of human gliomas at the single cell level. Our major goal was to define the different patterns of intratumoral clonal evolution that could contribute to the identification of those genetic profiles involved in determining the histopathological features of the tumor.

\section{Materials and methods}

\section{Patients and samples}

In the present study, tumors from 90 patients -46 males $(51 \%)$ and 44 females $(49 \%)$ - diagnosed with gliomas at the Neurosurgery Service of the University Hospital of Coimbra (Portugal), were analyzed. Mean age at diagnosis was $58 \pm 16$ years (range, 21 to 84 years). In all tumors, histological diagnosis and classification were performed according to the WHO criteria [3] with the following distribution: 72 cases $(80 \%)$ were astrocytomas (three grade I pilocytic astrocytomas, six were grade II diffuse astrocytomas, two corresponded to grade III anaplastic astrocytomas, 61 were grade IV primary or de novo glioblastomas, and two corresponded to grade IV gliosarcomas), 12 cases (13\%) corresponded to oligodendrogliomas (four grade II oligodendrogliomas and eight grade III anaplastic oligodendrogliomas), four cases (4\%) were oligoastrocytomas (one grade II oligoastrocytoma and three grade III anaplastic oligoastrocytomas), and, finally, two cases (2\%) were diagnosed as ependymomas (one grade II ependymoma and one grade III anaplastic ependymoma).

All samples were obtained after informed consent was given by the patient and the study was approved by the Ethics Committee of the University Hospital of Coimbra. In all cases, tumor samples were obtained by conventional surgical procedures and cut into several parts. Only those tumor tissue samples shown by gross and histologic inspection to be mostly or wholly tumor were used to prepare single cell suspensions for further iFISH studies. Preparation of single cell suspensions was prospectively done by mechanical disaggregation of fresh tumor tissue samples. Tissue areas mirror-cut to those used for iFISH analysis of single cell suspensions showed always $>65 \%$ of infiltrating tumor cells; the remaining cells corresponded to dead cells and eventually a few proportion of normal brain and leukocyte contamination $(<5 \%)$, as assessed by microscopical analysis of hematoxylin and eosin-stained tissue specimens. Once obtained, single tumor cell suspensions were stored at $-20^{\circ} \mathrm{C}$ until further processed. The remaining tumor sample was fixed in formalin and embedded in paraffin.

For each case, information about the histological characteristics of the tumor as well as the most relevant clinical and biological data (e.g., age, gender, and tumor localization) was recorded. 
Interphase fluorescence in situ hybridization studies

Thirteen genes (TP73, ANGPTL1, EGFR, ELN, TES, p16, ABL1, PTEN, Rb1, TP53, ZNF44, GLTSCR1, and BCR) and three chromosome centromeres (chromosomes 7, 9, and 10) were tested by iFISH (Supplementary Table 1). In all cases, iFISH analyses were performed on freshly frozen tumor cells after fixation in 3/1 methanol/acetic ( $v / v$; Panreac, Barcelona, Spain) with the following commercially available probesall obtained from Vysis, Inc. (Downers Grove, IL), except the 7p12 (EGFR) /alphasatellite 7 DNA dual-color probe that was obtained from Q-BIOgene (Carlsbad, CA)—-which were systematically used in double-stainings for the detection of numerical abnormalities of 16 different chromosome regions (please see Supplementary Table 1 for more detailed information about the probes used): (1) for chromosome 1, the LSI 1p36/LSI 1q25 dual-color probe was used; (2) for chromosome 19, the LSI 19q13/LSI 19p13 dual-color probe was employed; (3) for chromosome 7, the LSI ELN/LSI 7q31 dual-color probe was applied; (4) for chromosomes 9 and 22, the LSI bcr/abl ES dual-color probe was used; (5) in addition, also for chromosome 9, the LSI 9p21/CEP-9 dualcolor probe was used; and (6) for chromosome 10, the LSI PTEN/CEP-10 dual-color probe, was employed. For chromosomes 13 and 17, the LSI $R b 1$ and LSI p53 probes conjugated with spectrum orange (SO) were used, respectively. In order to accurately define the exact abnormalities carried by different tumor cell clones within a tumor sample, further appropriate multicolor stainings simultaneously using up to three different probes were performed whenever required, as illustrated in Fig. 1.

Briefly, fixed tumor cells were dropped onto cleaned, polyL-lysine-coated slides (Sigma, St. Louis, MO). The slides were then sequentially incubated for $10 \mathrm{~min}$ at $37^{\circ} \mathrm{C}$ with a solution containing $0.1 \mathrm{mg} / \mathrm{ml}$ of pepsin (Sigma), fixed in $1 \%$ acid-free formaldehyde $\left(\mathrm{CH}_{2} \mathrm{O}\right.$; Merck, Darmstadt, Germany) in phosphate-buffered saline for $10 \mathrm{~min}$ at room temperature and dehydrated in increasing concentrations of ethanol (Merck) in water $(70 \%, 90 \%$, and $100 \%)$. Once dried, the slides containing both the cells' DNA and the probes' DNA were denaturated $\left(6 \mathrm{~min}\right.$ at $\left.75^{\circ} \mathrm{C}\right)$ and subsequently hybridized overnight at $38^{\circ} \mathrm{C}$ in an Hybridizer thermocycler (Dako, Glostrup, Denmark). Once hybridized, slides were sequentially washed in $50 \%$ formamide (Merck) in a $2 \times$ saline sodium citrate (SSC) buffer $\left(10 \mathrm{~min}\right.$ at $\left.46^{\circ} \mathrm{C}\right)$ and in $2 \times \mathrm{SSC}(5 \mathrm{~min}$ at $46^{\circ} \mathrm{C}$ ). Finally, tumor cell nuclei were counterstained with $75 \mu \mathrm{l}$ of a solution containing 4,6-diamidino-2-phenylindole $(0.1 \mu \mathrm{g} / \mathrm{mL})$ (Sigma) and Vectashield (Vector Laboratories, Inc., Burlingame, CA), as anti-fading agent.

An Axioscope fluorescence microscope equipped with a $\times 100$ oil objective (Zeiss, Göttingen, Germany) was used for counting the number of hybridization spots per nuclei $(\geq 200$ nuclei/slide). Only those spots with a similar size, intensity and shape in non-overlapping nuclei, were counted; doublet signals were considered as single spots. Cut-offs used to define the presence of numerical abnormalities for each individual chromosome, were based on the analysis of normal human tissue samples, as previously described in detail [31]. Briefly, gains and losses of specific chromosome regions were considered to occur when $\geq 5 \%$ and $\geq 10 \%$ of the nuclei showed an increased and decreased number of fluorescent signals (spots) with respect to normal diploid cells, respectively. Specimens were considered to carry amplification of the EGFR when more than $10 \%$ of the tumor cells exhibited either an EGFR:CEP7 ratio $>2$ or multiple tight clusters of hybridization signals for the EGFR gene probe. Definition of the exact tumor cell clones present in each sample was based on the presence of nuclei carrying identical numbers of hybridization spots for all probes analyzed in that tumor sample. In this study, only those groups of cells showing identical cytogenetic profiles which represented $\geq 5 \%$ of the tumor cells in a sample were considered to be a clone [31]. The ancestral tumor cell clone was identified as that tumor cell clone which carried genetic abnormalities common to all other tumor cell clones. In turn, groups of cells carrying cytogenetic abnormalities not shared by all cytogenetic altered tumor cells were considered as secondary clones.

\section{Statistical methods}

In order to assess the statistical significance of the differences observed between groups of patients, the Student $t$ and the Mann-Whitney $U$ tests were used for parametric and nonparametric continuous variables, respectively; for categorical variables, the $X^{2}$ test was applied (SPSS software, SPSS 12.0, SPSS Inc, Chicago, IL). Overall survival curves were plotted according to the method of Kaplan-Meier and they were compared using the log-rank test. $p$ values $\leq 0.05$ were considered to be associated with statistical significance.

\section{Results}

In this study we applied iFISH for the analysis of intratumoral cytogenetic heterogeneity at the single cell level. This approach, allowed the identification within individual samples of distinct cytogenetic profiles. In virtually every case, one or more chromosomal abnormalities were shared by all clones in the sample while others were identified in only part of all tumor cells suggesting they could correspond to earlier vs later chromosomal changes, respectively (Fig. 1).

Frequency of numerical chromosome changes

Genetic abnormalities for at least one of the 16 different chromosome probes analyzed (1p36, 1q25, 7p12, 7q11, 


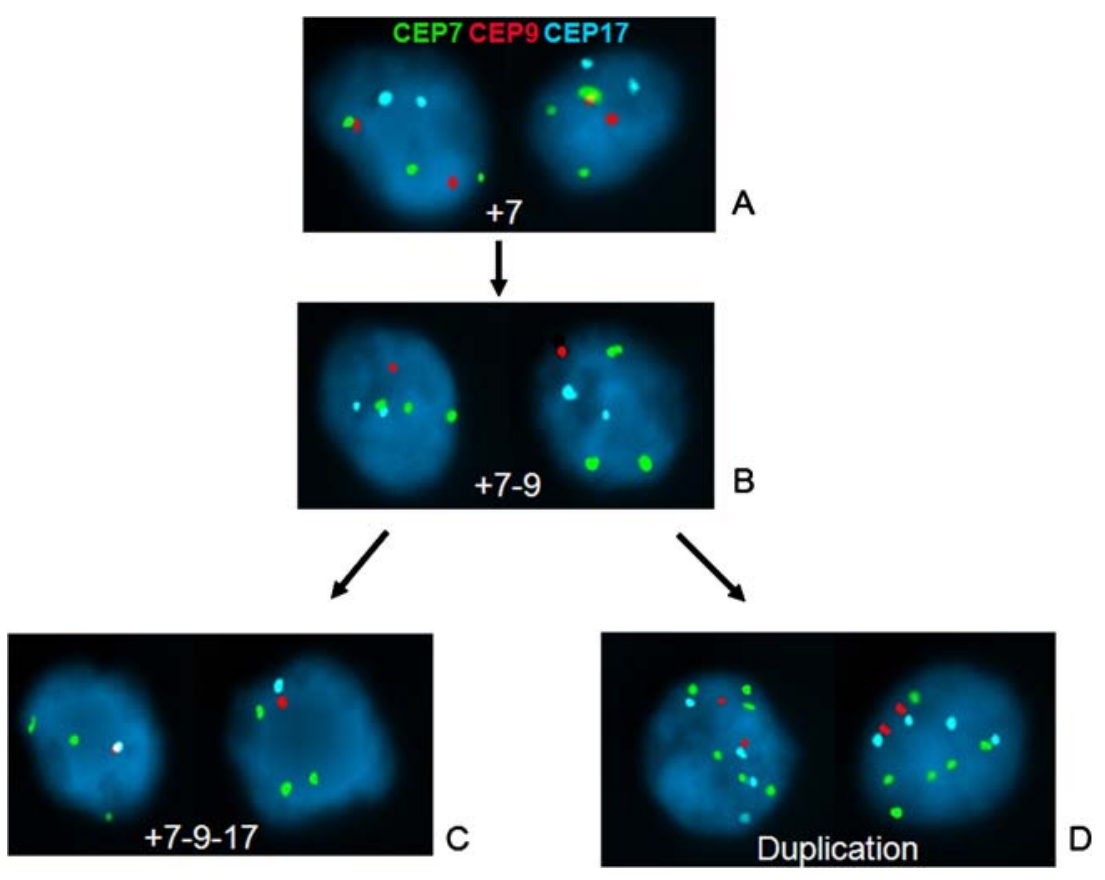

Fig. 1 Illustrating example of how different tumor cell clones were defined in a glioma using a three-color iFISH staining with probes directed against the centromeric regions of chromosomes 7, 9, and 17. As illustrated all nuclei counterstained in blue show gains of chromosome 7 (green small dots) while this was the only abnormality for the two nuclei displayed in a it was associated with loss of one copy of chromosome 9 (red dots) in the four nuclei shown in $\mathbf{b}$ and $\mathbf{c}$;

7q31, 9p21, 9q34, 10q23, 13q14, 17p13, 19p13, 19q13, and 22q11 locus and centromeres of chromosomes 7, 9, and 10) were systematically detected among the 90 gliomas studied. Overall, chromosome gains were more frequently observed than chromosome losses (68\% vs $51 \%$ of all numerical chromosome abnormalities detected) and included all type of polysomies with the presence of three to nine iFISH signals/cell for individual chromosomes; gene amplification was only detected for the EGFR gene at chromosome 7p12 (Fig. 2a). Regarding individual chromosomes, chromosomes 7 and $9 \mathrm{q} 34$ were the most frequently altered, and they were gained (including amplification of the EGFR gene) in $91 \%$ and $79 \%$ of the tumors, respectively. Interestingly, gains of chromosome 7 were found to be associated with deletion of the $7 \mathrm{p} 12$ chromosome region in six cases $(7 \%)$, del(7q11) in $12(13 \%)$, del (7q31) in 16 cases $(18 \%)$, and loss of centromere 7 in nine cases $(10 \%)$. In contrast, gains of chromosome $9 \mathrm{q} 34$ were frequently associated with hemizygous/homozygous deletion of the $9 p 21$ region. Del(9p21) together with del(10q23) corresponded to the most frequent chromosome losses and they were identified in around $80 \%$ of all cases $(77 \%$ and $78 \%$, respectively). The frequency of these and other numerical chromosomal abnormalities detected is detailed in Fig. 2a. in this latter panel the two nuclei displayed also show loss of one blue spot (monosomy 17). In turn, the two nuclei in $\mathbf{d}$ show duplication of the fluorescence signals of all three chromosomes analyzed with six, two, and four copies of chromosomes 7, 9, and 17, respectively. Arrows indicate the hypothetical model of clonal evolution established for this tumor based on the abnormalities detected with the three chromosome probes

Intratumoral cytogenetic heterogeneity and hypothetical pathways of clonal evolution

All except one tumor (99\%) showed two or more different tumor cell clones (number of tumor cells/clone of 5\% to 90\%) as defined by the iFISH patterns of cytogenetic abnormalities detected (Table 1). Presence of two clones was detected in five cases $(6 \%)$, three clones in $17(19 \%)$, four in 20 tumors (22\%), five in $17(19 \%)$, and $\geq 6$ tumor cell clones were found in 30 patients (33\%; Table 1). All glioma grades included tumors which displayed up to four different tumor cell clones, whereas five and six clones were only observed among high-grade tumors, with the exception of one grade II glioma (Table 1).

In $61 / 90$ cases (68\%), the cytogenetic aberrations found in common to all altered tumor cells consisted of abnormalities of either chromosome $7(43 \%)$ or the short arm of chromosome $9(16 \%)$ or both $(9 \%$; Fig. 2 b). Accordingly, amplification of the EGFR gene was found to be present in all altered tumor cells (Fig. 3d) of $22 \%$ of the cases, while three to five copies of chromosome 7 (Fig. 3a-c) were found in all tumor cell clones of 19/90 (21\%) cases (Fig. 2b). Isolated loss of either one or two copies of the 9p21 chromosome region (Fig. 3e, f) was also found to be shared by all tumor cell clones of 14/90 gliomas (16\%; Fig. 2b). 
Fig. 2 Overall frequency of different gains and losses of specific chromosome regions detected in glioma tumors (a) and distribution of the tumors according to the cytogenetic profile of the ancestral tumor cell clone $(\mathbf{b} ; n=90)$. Different tumors presented with distinct genetic alterations; in some cases a mixture of different abnormalities for the same chromosome was detected. In addition, it should be emphasized that low-grade astrocytomas $(n=9)$ typically showed gains for all chromosomes analyzed, which were associated with a tetraploid ancestral tumor cell clone among the later cases (see Table 3); in contrast, lowgrade oligodendrogliomas typically showed simultaneous 1 osses for chromosome $1 \mathrm{p}$ and $19 \mathrm{q}(n=3 / 4$ cases $)$ a
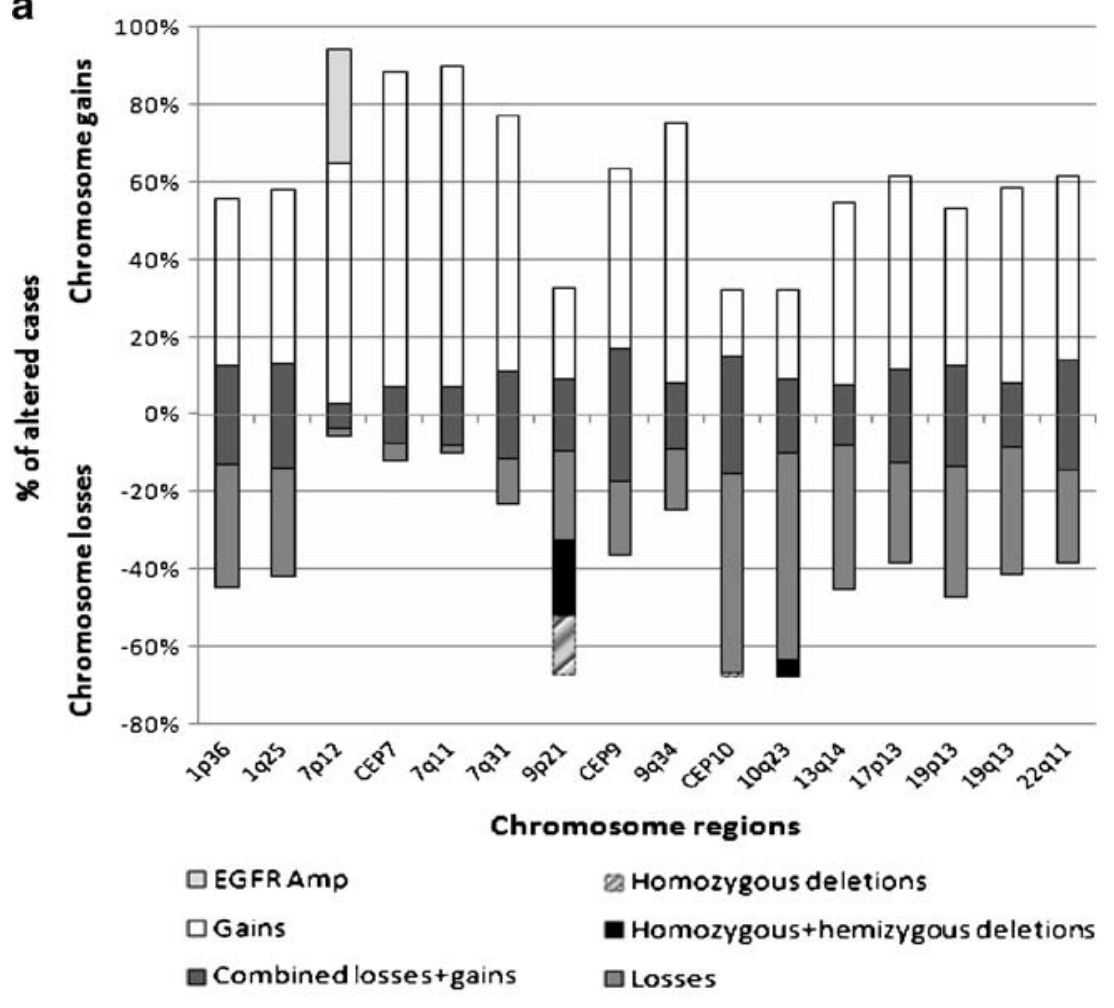

b

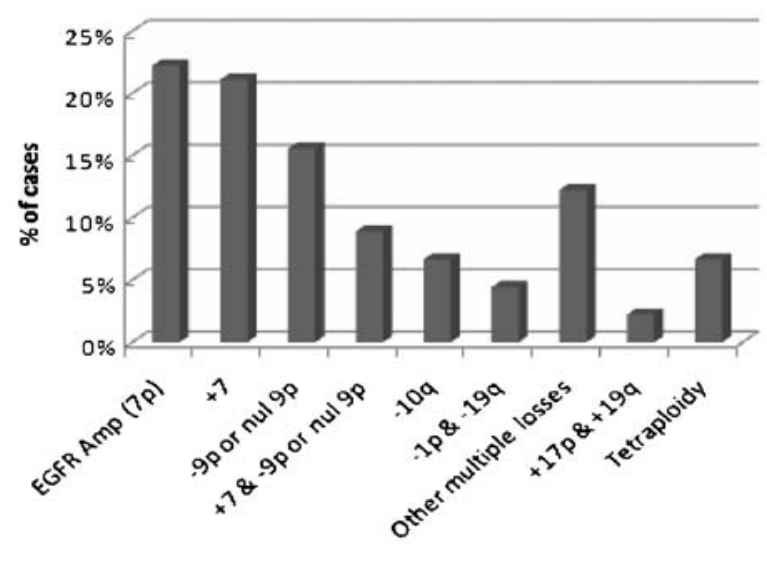

Ancestral tumor cell clone
Other cytogenetic aberrations found at lower frequencies in all tumor cell clones from individual cases included gains of chromosome 7 in association with hemizygous/homozygous deletion of the $9 \mathrm{p} 21$ chromosome region ( $9 \%$ of the cases), losses of chromosome $10 \mathrm{q}$ ( $7 \%$ of the cases), combined 1p36/19q13 losses (4\%), coexisting deletions of several chromosome regions (12\%), and coexisting gains of the 17 p13 and $19 q 13$ chromosome regions (2\%); in $7 \%$ of the cases the ancestral tumor cell clones were tetraploid, with several additional changes being found in some of these tumors (Fig. 2b).

Detailed analysis of the different neoplastic cell clones present in each glioma tumor pointed out the occurrence of variable and complex patterns of intratumoral clonal evolution, as schematically illustrated in Fig. 4 and Supplementary Fig. 1. As mentioned above, in most cases $(67 / 90 ; 74 \%)$, numerical chromosomal abnormalities common to all altered cells from individual tumors involved chromosomes 7, 9, and/or 10, for a total of five of nine different cytogenetic profiles (Fig. 4 and Supplementary Fig. 1). In the other tumors $(23 / 90 ; 26 \%)$, common abnormalities to all genetically altered cells consisted of combined loss of the $1 \mathrm{p} 36$ and $19 \mathrm{q} 13$ chromosome regions $(4 / 90 ; 4 \%)$, simultaneous losses of several chromosome regions $(11 / 90 ; 12 \%)$, simultaneous gains of $17 \mathrm{p} 13$ and $19 \mathrm{q} 13(2 / 90 ; 2 \%)$ or a tetraploid karyotype (6/90 cases; 7\%) (Fig. 4). Additional cytogenetic changes to those listed above and shared by all altered cells were highly variable 
Table 1 Glioma tumors $(n=90)$ : number of cytogenetically defined tumor cell clones according to the WHO tumor grade

\begin{tabular}{|c|c|c|c|c|c|}
\hline \multirow[t]{2}{*}{ No. of clones/tumor } & \multicolumn{4}{|c|}{ Tumor grade* } & \multirow[t]{2}{*}{ Total cases } \\
\hline & $\mathrm{I}(n=3)$ & II $(n=12)$ & III $(n=14)$ & IV $(n=61)$ & \\
\hline 1 & $0 / 3(0 \%)$ & $1 / 12(8 \%)$ & $0 / 14(0 \%)$ & $0 / 61(0 \%)$ & $1 / 90(1 \%)$ \\
\hline 2 & $2 / 3(67 \%)$ & $2 / 12(17 \%)$ & $1 / 14(7 \%)$ & $0 / 61(0 \%)$ & $5 / 90(6 \%)$ \\
\hline 3 & $1 / 3(33 \%)$ & $5 / 12(42 \%)$ & $6 / 14(43 \%)$ & $5 / 61(8 \%)$ & 17/90 (19\%) \\
\hline 4 & $0 / 3(0 \%)$ & $3 / 12(25 \%)$ & $2 / 14(14 \%)$ & $15 / 61(25 \%)$ & $20 / 90(22 \%)$ \\
\hline 5 & $0 / 3(0 \%)$ & $0 / 12(0 \%)$ & $1 / 14(7 \%)$ & $16 / 61(26 \%)$ & $17 / 90(19 \%)$ \\
\hline$\geq 6$ & $0 / 3(0 \%)$ & $1 / 12(8 \%)$ & $4 / 14(29 \%)$ & $25 / 61(41 \%)$ & $30 / 90(33 \%)$ \\
\hline
\end{tabular}

Results expressed as number of cases/total cases and percentage in brackets

WHO World Health Organization

$* p<0.001$

and heterogeneous. Accordingly, as illustrated in more detail in Supplementary Fig. 1, cases with amplification of the EGFR gene (20 cases; 22\%) systematically displayed further gains of $7 \mathrm{q}$ (10/20 cases) and/or losses of the 10q23 (14/20 cases) in association or not with losses of one or two copies of the 9p21 region (16/20 cases) and/or loss of 1 p36 chromosome region $(6 / 20$ cases). In turn, those tumors which showed gains of chromosome 7 in common to all tumor cell clones typically showed the presence of del (10q23) (5/19 cases) and/or $\operatorname{del}(9 \mathrm{p})$ (2/19 cases) in association with variable patterns of other chromosome changes, except for six cases which had tetraploid cells (Fig. 4 and Supplementary Fig. 1). Cases with all tumor cell clones found to show isolated gains of $+17 p /+19 q$ displayed distinct patterns of acquisition of additional chromosome gains and losses in each of the clones (Fig. 4 and Supplementary Fig. 1).

Of note, those cases which had deletions of chromosome $9 p$ in association or not with gains of chromosome 7 in common to all altered tumor cells, displayed cytogenetic
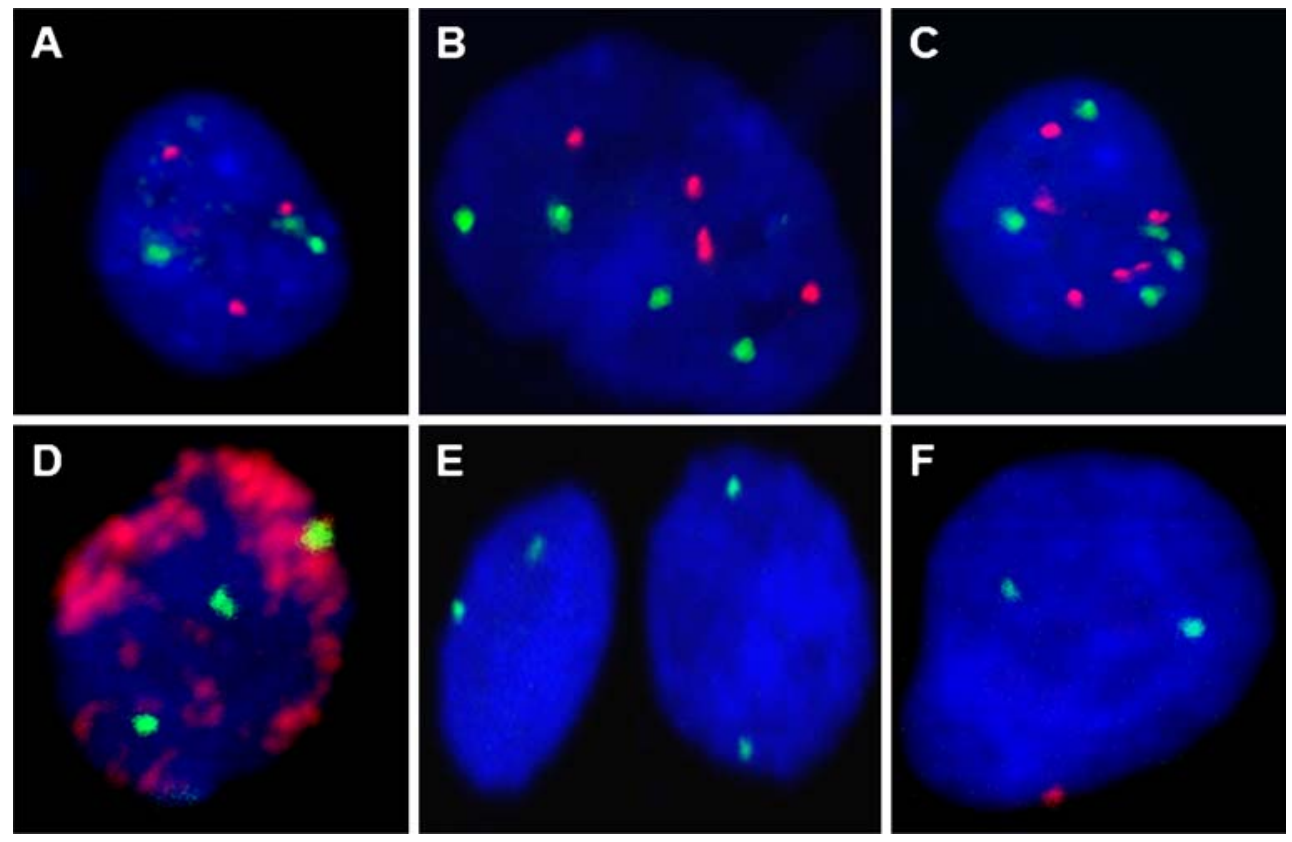

Fig. 3 Illustrating examples of some of the most frequently detected chromosomal abnormalities identified in glioma tumor cells by interphase fluorescence in situ hybridization (iFISH); original magnification $\times 1,000$. In a-c, nuclei (blue background signal) corresponding to tumors showing gains - trisomy (a), tetrasomy (b), and pentasomy (c) - of chromosome 7 (red spots) are displayed; in these panels (a to c) green spots correspond to a chromosome 7 centromeric probe. Amplification of the EGFR locus (7p12) was found in 20 gliomas (red spots) and it is illustrated in (d) where green signals also correspond to the fluorescence emissions associated with a centromeric probe for chromosome 7. e and f show illustrating examples of two and one tumor cell carrying nul(9p21) and del(9p21), as revealed by the absence of red spots or the presence of a single red spot, respectively; these three cells also show two green spots each, corresponding to a probe directed to the centromeric region of chromosome 9 


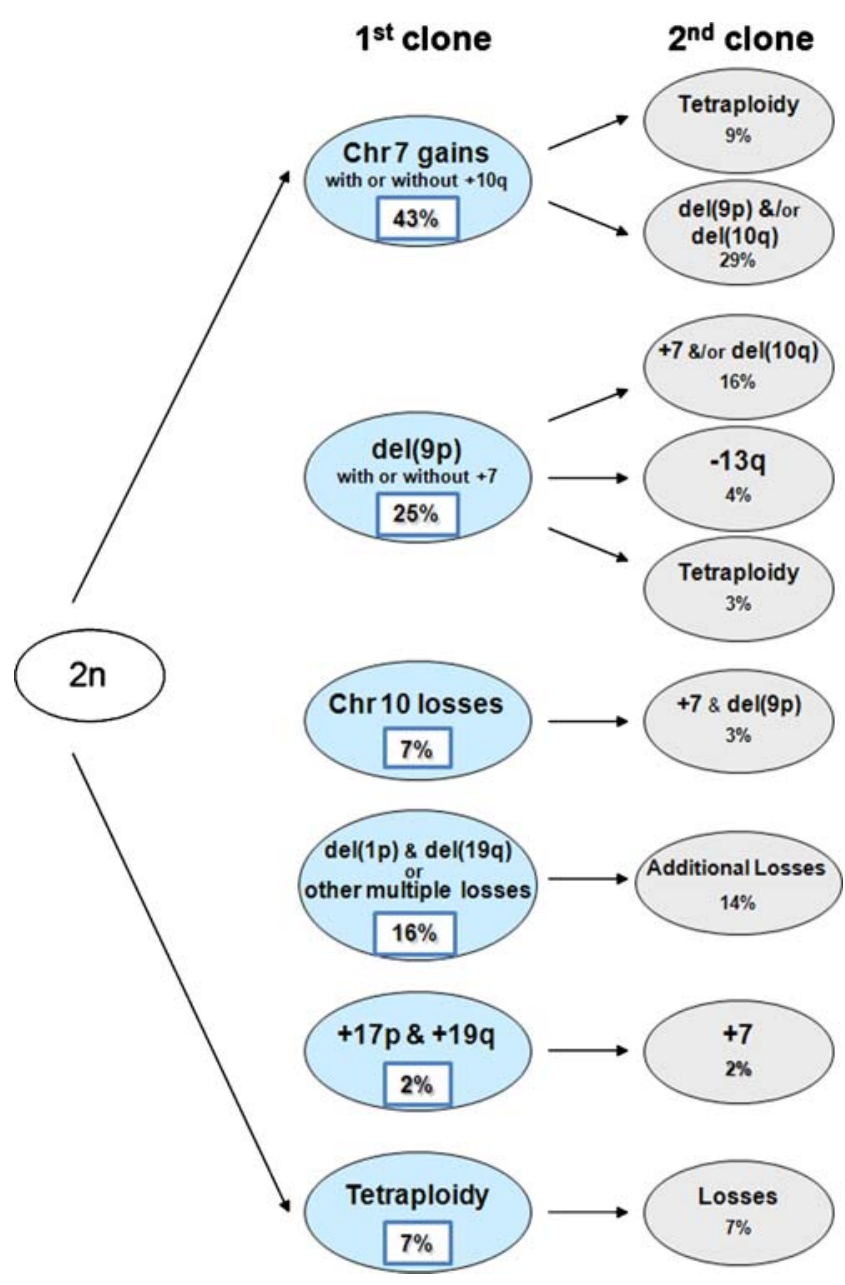

Fig. 4 Hypothetical pathways of intratumoral clonal evolution of gliomas $(n=90)$ defined on the basis of the most frequent cytogenetic patterns detected for the 16 different chromosome probes analyzed. In this figure, only the most frequently detected ancestral tumor cell clones and their corresponding subsequent second clones are displayed. Noteworthy, most (five of six tumors) tetraploid cases corresponded to low-grade astrocytomas and around half of all tumors showing $\operatorname{del}(1 \mathrm{p}) / \operatorname{del}(19 q)$ and/or other multiple losses in their ancestral tumor cell clone were low-grade gliomas (two diffuse astrocytomas, four oligodendrogliomas, and one ependimoma out of 15 cases); in contrast, high-grade gliomas predominated among the other cytogenetic groups. For more detailed information on the exact cytogenetics profiles of each clone please see Supplementary Fig. 1

patterns with between one and four additional abnormalities of chromosomes 1p, 7, 10q, 13q, and $17 \mathrm{p}$; in addition, $3 \%$ of these cases with del(9p21) with or without gains of chromosome 7 in common to all tumor cell clones, showed tetraploidization (Fig. 4 and Supplementary Fig. 1). As it may be inferred from what is describe above, tetraploidization with variable losses and gains of one or more chromosomes was a relatively common finding and it was detected in secondary clones of around half of the cases $(47 / 90 ; 52 \%)$, in addition to those cases $(7 \%)$ that presented tetraploidy in common to all tumor cell clones (Fig. 4 and

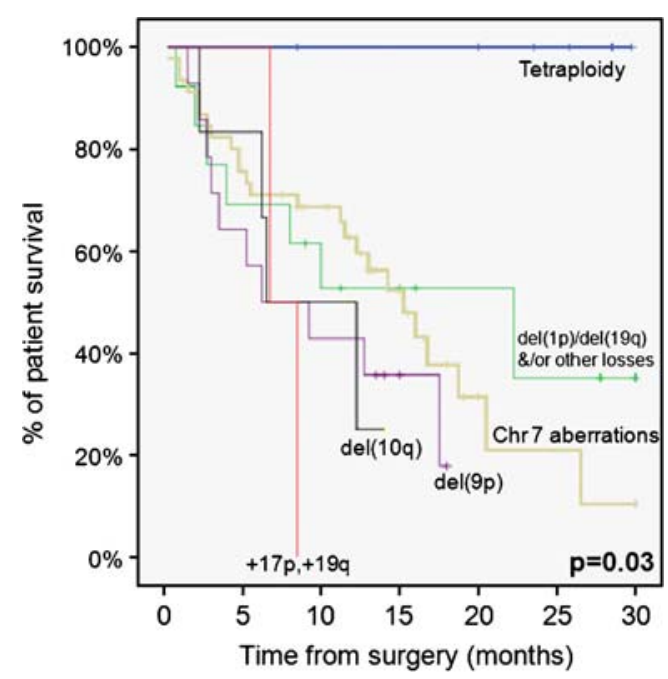

Fig. 5 Overall survival curves of gliomas grouped according to the cytogenetic abnormalities found to be common to all altered tumor cells. Of note, most (five of six tumors) tetraploid cases corresponded to low-grade astrocytomas and around half of all tumors showing del (1p)/del(19q) and/or other multiple losses in their ancestral tumor cell clone were low-grade gliomas (two diffuse astrocytomas, four oligodendrogliomas, and one ependimoma out of 15 cases); in contrast, high-grade gliomas predominated among the other cytogenetic groups

Supplementary Fig. 1). However, in some subgroups such as cases with combined del(1p) and del(19q), secondary clones showed multiple additional chromosome losses in the absence of a tetraploid karyotype (Fig. 4 and Supplementary Fig. 1)

Interestingly, once gliomas were grouped according to the cytogenetic profile found to be common to all altered tumor cells, a longer overall survival was observed $(p=0.03)$ for cases that displayed either tetraploidy or del(1p) associated with del $(19 q)$ compared to patients who had abnormalities of chromosome $7, \operatorname{del}(9 p)$, del(10q) and simultaneous gains of chromosome 17p and 19q (Fig. 5).

Cytogenetic profiles and tumor grade

Upon analyzing the frequency of the distinct cytogenetic abnormalities detected in gliomas according to tumor histopathology, several remarkable differences were noted between astrocytic and oligodendroglial, as well as between grade I/II and grade III/IV cases (Table 2). Accordingly, astrocytic grade I/II tumors ( $9 / 72$ cases; $13 \%$ ) were mainly characterized by the presence of gains for all chromosome regions analyzed (including all three astrocytic grade I tumors) consistent with tetraploidy in five of nine cases. In contrast, high-grade astrocytic tumors (63/72 tumors; $88 \%$ ) showed more complex and heterogeneous genetic alterations, with a significantly higher frequency of chromosome abnormalities (Table 2). 


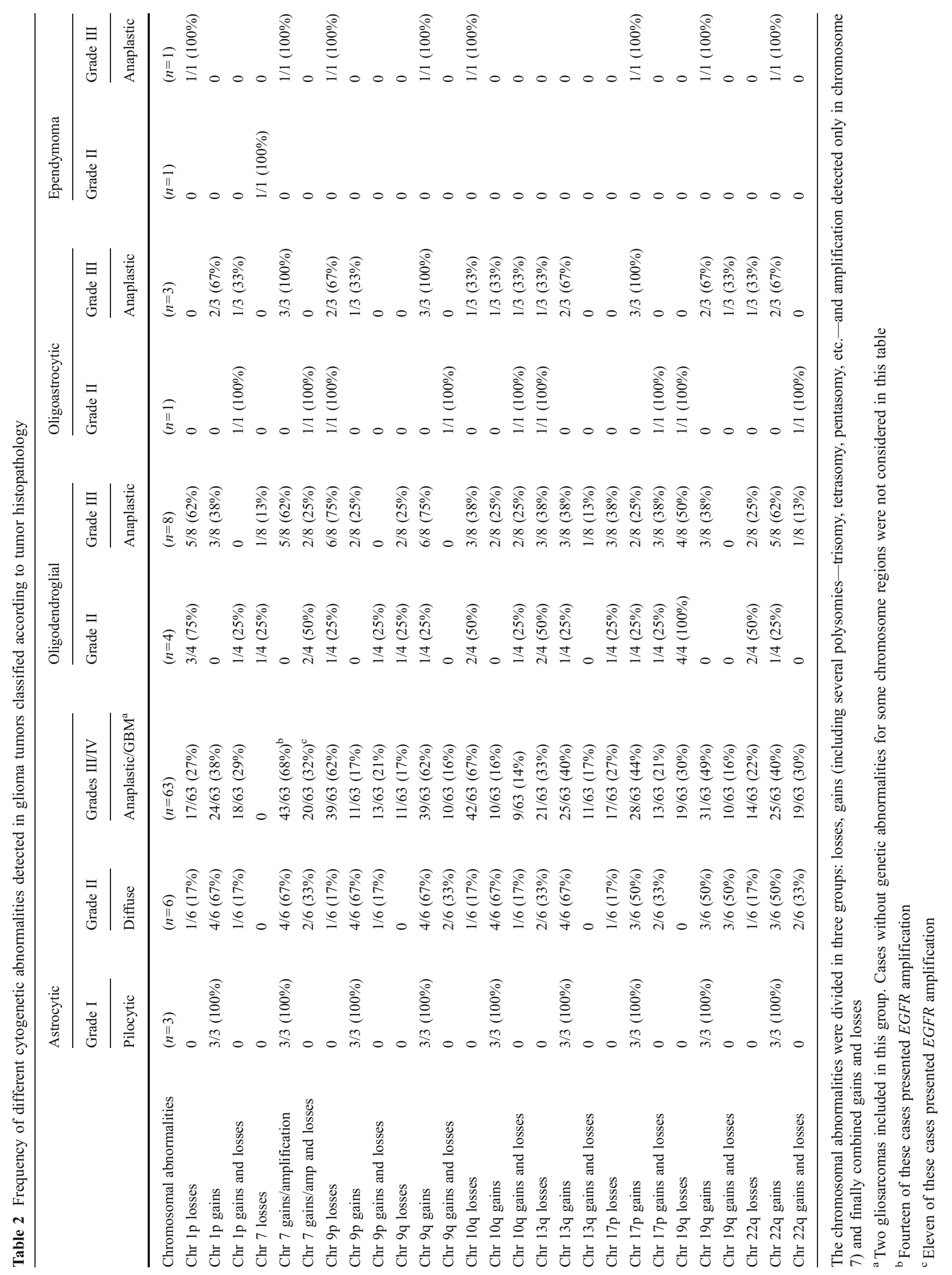


Interestingly, a significant association was also found between tumor grade and the iFISH patterns of cytogenetic abnormalities detected in common to all tumor cell clones identified in individual cases (Table 3). Accordingly, while grade I/II astrocytic tumors showed either gains of chromosome 7 or tetraploidy in common to all altered tumor cells, high-grade astrocytic tumors (grades III/IV) revealed more complex cytogenetic patterns, with a considerable lower frequency of tetraploid tumors (Table 3). Although the number of cases analyzed for other histopathological subtypes was rather limited, combined loss of the $1 \mathrm{p} 36$ and $19 \mathrm{q} 13$ regions was most frequently found in common to all tumor cell clones in oligodendrogliomas (4/12 cases; $33 \%$, particularly in low-grade cases, three of four cases). Despite this, as found in high-grade oligoastrocytic tumors, anaplastic oligodendrogliomas, in addition to $1 \mathrm{p} 36 / 19 \mathrm{q} 13$ losses, showed more heterogeneous cytogenetic patterns in common to all tumor cell clones with respect to oligodendroglial grade II tumors; similarly to what was observed among high-grade astrocytomas, these included gains of chromosome 7, losses of the 9p21 chromosome region and other multiple chromosome losses (Table 3).

\section{Discussion}

Until now, few studies have been reported in which the cytogenetic heterogeneity of glioma tumors is assessed at the intratumoral cell level [32, 33], and only Lopez-Gines et al. [34] and Wemmert et al. [33] have used iFISH analyses based on a limited number of chromosome probes, to assess clonal evolution in glioblastomas and pilocytic astrocytomas, respectively. Through the study of 25 glioblastomas LopezGines et al. [34] suggested that trisomy/polysomy 7 and monosomy 10 were frequently associated in glioblastomas, whereas EGFR amplification could represent a later event not associated with the former profile (gains of chromosome 7/chromosome 10 losses). In turn, Wemmert et al. [33] confirmed the occurrence of intratumoral cytogenetic heterogeneity among 14 pilocytic astrocytomas, through iFISH analysis of numerical abnormalities for chromosomes 7,8 , 17, and the TP53 gene.

In this study, we used a multicolor iFISH approach aimed at the investigation of a large number $(n=16)$ of different chromosome regions, including those more frequently altered in gliomas, to assess intratumoral genetic heterogeneity in a series of 90 patients studied at diagnosis. In order to identify the exact sequence of accumulation of chromosomal abnormalities in individual tumors carrying multiple subclones, we assumed that karyotypic abnormalities shared by all subclones should represent relatively early changes; in contrast, later cytogenetic changes would only be present in some of the

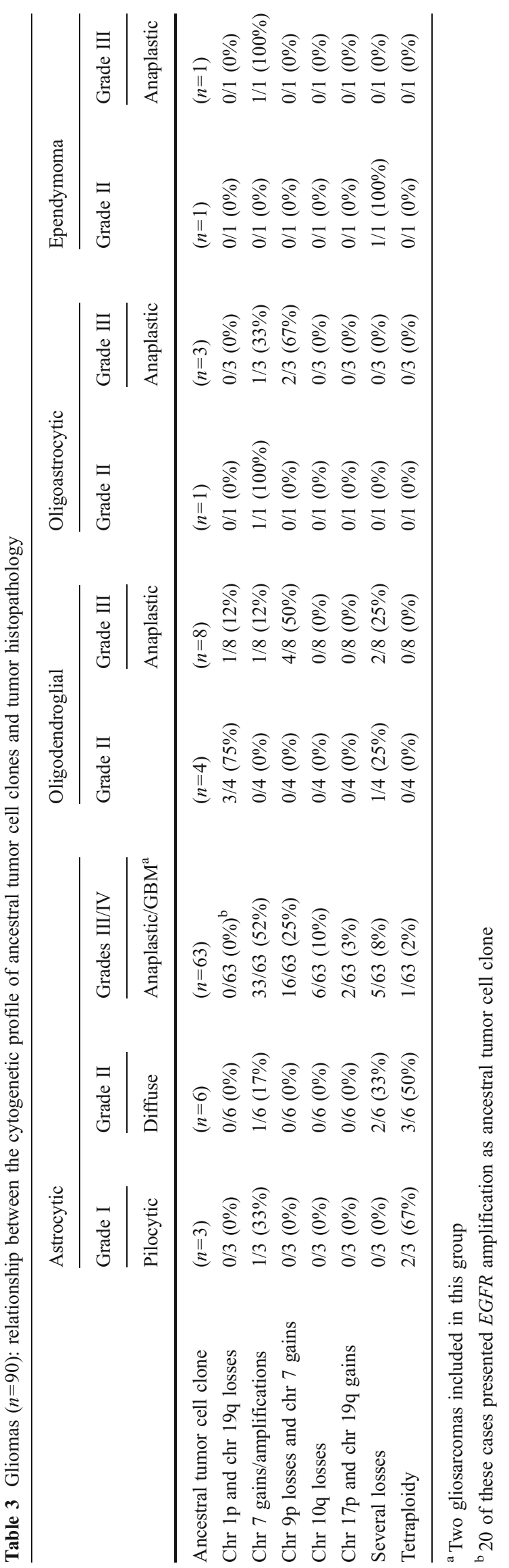


other tumor cell clones. In line with this concept of clonal evolution, our results show that in virtually all gliomas, $\geq 2$ cytogenetically related tumor cell clones which carry different chromosomal abnormalities, are present.

Overall, nine different cytogenetic profiles were identified in the ancestral tumor cell clones of the 90 cases analyzed. These most commonly involved partial or complete gains of chromosome 7-including amplification of the EGFR gene-hemizygous or homozygous deletion of 9 p21 and del(10q); interestingly, combinations of such alterations were either already detected in the ancestral tumor cell clone or acquired in subsequent, highly represented clones. Of note, these complex cytogenetic patterns typically occurred prior to tetraploidization-a cytogenetic profile detected during evolution of a great proportion of all tumors analyzed-in the context of a relatively high number of tumor cell clones/sample and they were characteristic of high-grade astrocytic tumors.

Altogether, these results clearly show that complex karyotypes in which combined gains of chromosome 7including amplification of the EGFR gene- $\operatorname{del}(9 \mathrm{p} 21)$ and/or $\operatorname{del}(10 q 23)$ coexist, are crucial events for the development of a great majority of gliomas with an astrocytic phenotype; in addition, they support previous observations about the role of combined alterations of the EGFR, p16/p14 ${ }^{A R F}$, and/or PTEN genes in the malignant (high-grade) transformation of these tumors $[10,24,35,36]$. In line with this, it has been previously shown that most gliomas coexpress the EGFR and $E G F$ molecules, which are involved in an autocrine loop of growth stimulation [37]. Of note, EGFR mediates its cellular effects through the PI3K-Akt pathway [38], which is repressed by the expression of PTEN [39, 40]; loss/mutation of this later gene results in a direct increase in cell growth together with a blockade of apoptosis and up-regulation of vascular endothelial growth factor production [37, 40, 41]. Similarly, deletion of $p 16$ and $p 14^{A R F}$ would also favor cell proliferation in these tumors, particularly when associated with mutations or epigenetic silencing of the remaining copy of these genes $[40,42]$. Such a cytogenetic profile consisting of disruption of the $p 16-p R b-p 14^{A R F}-T P 53$ cell cycle control pathways, inactivation of tumor suppressor genes on chromosome 10 (e.g. PTEN) and amplification of the EGFR gene, has been associated with high-grade gliomas with an astrocytic phenotype [40] as also found in our series. In turn, the higher number of tumor cell clones observed among high-grade gliomas suggests a high genetic/chromosomal instability, which would facilitate the emergence and sequential selection of more aggressive subclones that would lead to tumor progression and more aggressive histopathological features. Of note, this high genetic/chromosomal instability cannot be therapy-induced since the studied glioblastoma multiforme are primary (or de novo) GBM, without any previous treatment.
Other less frequent cytogenetic profiles recurrently observed in the ancestral tumor cell clones included tetraploidy, combined loss of 1p36/19q13 and combined gains of chromosomes 17 and 19; while the later pattern was only found in a few high-grade glioblastomas, the former were characteristic of low-grade tumors with a better outcome and an astrocytic or oligodendroglial/mixed phenotype, respectively. In contrast to high-grade gliomas, few cytogenetic profiles were detected in common to all altered tumor cells in grade I/II astrocytic tumors. Such profiles were consistent with tetraploidy and less frequently, with chromosome 7 gains in the absence of losses of chromosomes 9p21 and 10q23, immediately followed by tetraploidization in subsequent tumor cell clones. Based on these observations, it could be hypothesized that early occurrence of tetraploidization in association with duplication of the $B R A F$ gene in pylocytic astrocytomas [43, 44] could prevent histopathological grade I/II astrocytomas from evolving into an invasive phenotype and a highly progressive clinical behavior tied to aggressive histopathological features; this could be due to the fact that p16/p14 $4^{A R F}$ and chromosome $10 \mathrm{q}$ losses that occur after tetraploidization would have a more limited impact in the aggressiveness of the tumor because at least one copy of each allele of these genes would still be retained. In line with this, a tetraploidy checkpoint has been associated with prevention of tumor progression among gliomas [45, 46] and these cytogenetic profiles were also much less frequently observed among high-grade gliomas, where acquisition of multiple cytogenetic abnormalities leading to relatively complex cytogenetic profiles, typically preceded tetraploidization. Although early tetraploidization was associated with a significantly better clinical outcome, this could be due to its association with low-grade astrocytomas and deserves further multivariate analyses in larges series of patients to determine its potential independent prediction value.

Combined losses of 1p36 and 19q13, were detected in common to all tumor cells in $3 / 4(75 \%)$ grade II oligodendrogliomas, while only $1 / 8$ (12\%) high-grade oligodendrogliomas showed this cytogenetic profile in its ancestral tumor cell clone. At present, the molecular mechanisms involved in the ontogeny of oligodendroglial tumors remains largely unclear. However, recent reports point out the potential involvement of $p 21$ [47], and other recently identified oligodendroglioma-associated candidate tumor suppressor genes localized at the $1 \mathrm{p} 36$ and $19 \mathrm{q} 13$ chromosome regions, in the development of oligodendrogliomas [48, 49]. In fact, it has been shown that, del(1p) and $\operatorname{del}(19 q)$, as well as translocations involving these chromosome regions are the genetic hallmark of oligodendroglial tumors [50-53] as also found in our series; however, it should be noted that in line with our findings, losses of chromosomes $1 p$ and $19 q$ could be less commonly 
detected in anaplastic/grade III versus grade II oligodendrogliomas [3, 49].

Altogether, these results may suggest that while astrocytic and oligodendroglial tumors may progress $[27,28,54]$ from histologically low- to high-grade tumors, frequently, different genetic pathways could be involved in histologically lowversus high-grade gliomas [55]. In line with this hypothesis, previous studies aimed at dissecting the genetic pathways of tumor progression through the comparison of the karyotypes of different histological subgroups of gliomas [4, 9-11] have pointed out the existence of two clearly distinct cytogenetic patterns. One corresponds to the majority of cases representing primary or de novo glioblastomas, which are genetically characterized by loss/mutation of 10q/PTEN, EGFR amplification and/or $p 16$ deletion with a low frequency of TP53 mutations. The second cytogenetic pattern is characteristic of secondary glioblastomas, which develop through progression from low-grade diffuse astrocytomas or anaplastic astrocytomas where TP53 mutation is the most frequent and earliest detectable genetic alteration $[24,30]$. Our results support and extend on this hypothesis by showing that, at the intratumoral cell level, early tetraploidization together with combined 1p36/19q13 losses are associated with low-grade astrocytomas and oligodendroglial tumors, respectively; of note, tetraploidy was similarly found in pilocytic grade I as well as grade II, diffuse astrocytomas as also reported by others $[56,57]$. In contrast, the ancestral tumor cell clones of high-grade gliomas are more likely associated with early occurrence of combined gains/amplification of the EGFR gene in chromosome 7 , hemizygous or homozygous del (9p21) and/or deletion of the PTEN gene in chromosome $10 \mathrm{q}$, followed by acquisition of complex karyotypes prior to tetraploidization. Based on these observations, it could be hypothesized that early tetraploidization in gliomas with an astrocytic phenotype could prevent these tumors from having an aggressive behavior, even in the presence of TP53 mutation/deletion [40, 58, 59]; in contrast, occurrence of tetraploidization at relatively advanced stages of the disease, after gains of chromosome 7/EGFR amplification, del(9p21) and/or del(10q23) have already occurred, would not have such an impact. These observations together with the limited number of low-grade tumors included in our study point out the need to confirm our observations in larger series of lowgrade gliomas, in which comparative analysis of the cytogenetic profiles of primary versus secondary high-grade tumors are also analyzed.

Acknowledgments This work has been partially supported by grants from FCT (Portuguese Foundation for Science and Technology, PhD fellowships SFRH/BD/11820/2003 and SFRH/BD/23086/2005), FCG (Portuguese Calouste Gulbenkian Foundation, Project Ref. 68708) and Spanish Network of Cancer Research Centers (RD06/ 0020/0035; RTICC; Instituto de Salud Carlos III, Ministerio of Science and Innovation, Madrid, Spain).

\section{References}

1. Miller CR, Perry A (2007) Glioblastoma. Arch Pathol Lab Med 131:397-406

2. Ohgaki H, Kleihues P (2005) Epidemiology and etiology of gliomas. Acta Neuropathol 109:93-108

3. Louis DN, Ohgaki H, Wiestler OD, Cavenee WK (2007) WHO classification of tumours of the central nervous system. IARC, Lyon

4. Maher EA, Furnari FB, Bachoo RM, Rowitch DH, Louis DN et al (2001) Malignant glioma: genetics and biology of a grave matter. Genes Dev 15:1311-1333

5. Wolff JE, Berrak S, Koontz Webb SE, Zhang M (2008) Nitrosourea efficacy in high-grade glioma: a survival gain analysis summarizing 504 cohorts with 24193 patients. J Neurooncol 88:57-63

6. Stupp R, Mason WP, van den Bent MJ, Weller M, Fisher B et al (2005) Radiotherapy plus concomitant and adjuvant temozolomide for glioblastoma. N Engl J Med 352:987-996

7. Burton EC, Lamborn KR, Feuerstein BG, Prados M, Scott J et al (2002) Genetic aberrations defined by comparative genomic hybridization distinguish long-term from typical survivors of glioblastoma. Cancer Res 62:6205-6210

8. Fuller CE, Schmidt RE, Roth KA, Burger PC, Scheithauer BW et al (2003) Clinical utility of fluorescence in situ hybridization (FISH) in morphologically ambiguous gliomas with hybrid oligodendroglial/astrocytic features. J Neuropathol Exp Neurol 62:1118-1128

9. Phillips HS, Kharbanda S, Chen R, Forrest WF, Soriano RH et al (2006) Molecular subclasses of high-grade glioma predict prognosis, delineate a pattern of disease progression, and resemble stages in neurogenesis. Cancer Cell 9:157-173

10. Kramar F, Zemanova Z, Michalova K, Babicka L, Ransdorfova S et al (2007) Cytogenetic analyses in 81 patients with brain gliomas: correlation with clinical outcome and morphological data. J Neurooncol 84:201-211

11. Mizoguchi M, Betensky RA, Batchelor TT, Bernay DC, Louis DN et al (2006) Activation of STAT3, MAPK, and AKT in malignant astrocytic gliomas: correlation with EGFR status, tumor grade, and survival. J Neuropathol Exp Neurol 65:11811188

12. Homma T, Fukushima T, Vaccarella S, Yonekawa Y, Di Patre PL et al (2006) Correlation among pathology, genotype, and patient outcomes in glioblastoma. J Neuropathol Exp Neurol 65:846854

13. Korshunov A, Sycheva R, Golanov A (2005) The prognostic relevance of molecular alterations in glioblastomas for patients age $<50$ years. Cancer 104:825-832

14. Jeon YK, Park K, Park CK, Paek SH, Jung HW et al (2007) Chromosome $1 \mathrm{p}$ and $19 \mathrm{q}$ status and $\mathrm{p} 53$ and p16 expression patterns as prognostic indicators of oligodendroglial tumors: a clinicopathological study using fluorescence in situ hybridization. Neuropathology 27:10-20

15. Walker C, du Plessis DG, Joyce KA, Machell Y, Thomson-Hehir J et al (2003) Phenotype versus genotype in gliomas displaying inter- or intratumoral histological heterogeneity. Clin Cancer Res 9:4841-4851

16. Liang Y, Diehn M, Watson N, Bollen AW, Aldape KD et al (2005) Gene expression profiling reveals molecularly and clinically distinct subtypes of glioblastoma multiforme. Proc Natl Acad Sci U S A 102:5814-9

17. Nutt CL, Mani DR, Betensky RA, Tamayo P, Cairncross JG et al (2003) Gene expression-based classification of malignant gliomas correlates better with survival than histological classification. Cancer Res 63:1602-1607 
18. Koschny R, Koschny T, Froster UG, Krupp W, Zuber MA (2002) Comparative genomic hybridization in glioma: a meta-analysis of 509 cases. Cancer Genet Cytogenet 135:147-159

19. Wiltshire RN, Herndon JE 2nd, Lloyd A, Friedman HS, Bigner DD et al (2004) Comparative genomic hybridization analysis of astrocytomas: prognostic and diagnostic implications. J Mol Diagn 6:166-179

20. Fuller CE, Wang H, Zhang W, Fuller GN, Perry A (2002) Highthroughput molecular profiling of high-grade astrocytomas: the utility of fluorescence in situ hybridization on tissue microarrays (TMA-FISH). J Neuropathol Exp Neurol 61:1078-1084

21. Cowell JK, Matsui S, Wang YD, LaDuca J, Conroy J et al (2004) Application of bacterial artificial chromosome array-based comparative genomic hybridization and spectral karyotyping to the analysis of glioblastoma multiforme. Cancer Genet Cytogenet 151:36-51

22. Kamiryo T, Tada K, Shiraishi S, Shinojima N, Nakamura H et al (2002) Analysis of homozygous deletion of the p16 gene and correlation with survival in patients with glioblastoma multiforme. J Neurosurg 96:815-822

23. Okada Y, Hurwitz EE, Esposito JM, Brower MA, Nutt CL et al (2003) Selection pressures of TP53 mutation and microenvironmental location influence epidermal growth factor receptor gene amplification in human glioblastomas. Cancer Res 63:413416

24. Ueki K, Nishikawa R, Nakazato Y, Hirose T, Hirato J et al (2002) Correlation of histology and molecular genetic analysis of $1 \mathrm{p}$, 19q, 10q, TP53, EGFR, CDK4, and CDKN2A in 91 astrocytic and oligodendroglial tumors. Clin Cancer Res 8:196-201

25. Liu L, Ichimura K, Pettersson EH, Goike HM, Collins VP (2000) The complexity of the $7 \mathrm{p} 12$ amplicon in human astrocytic gliomas: detailed mapping of 246 tumors. J Neuropathol Exp Neurol 59:1087-1093

26. Backlund LM, Nilsson BR, Goike HM, Schmidt EE, Liu L et al (2003) Short postoperative survival for glioblastoma patients with a dysfunctional $\mathrm{Rb} 1$ pathway in combination with no wild-type PTEN. Clin Cancer Res 9:4151-4158

27. Ohgaki H, Dessen P, Jourde B, Horstmann S, Nishikawa T et al (2004) Genetic pathways to glioblastoma: a population-based study. Cancer Res 64:6892-6899

28. Ohgaki H, Kleihues P (2007) Genetic pathways to primary and secondary glioblastoma. Am J Pathol 170:1445-1453

29. Reifenberger J, Ring GU, Gies U, Cobbers L, Oberstrass J et al (1996) Analysis of p53 mutation and epidermal growth factor receptor amplification in recurrent gliomas with malignant progression. J Neuropathol Exp Neurol 55:822-831

30. Fulci G, Ishii N, Maurici D, Gernert KM, Hainaut P et al (2002) Initiation of human astrocytoma by clonal evolution of cells with progressive loss of p53 functions in a patient with a $283 \mathrm{H}$ TP53 germ-line mutation: evidence for a precursor lesion. Cancer Res 62:2897-2905

31. Sayagues JM, Tabernero MD, Maillo A, Espinosa A, Rasillo A et al (2004) Intratumoral patterns of clonal evolution in meningiomas as defined by multicolor interphase fluorescence in situ hybridization (FISH): is there a relationship between histopathologically benign and atypical/anaplastic lesions? J Mol Diagn 6:316-325

32. Harada K, Nishizaki T, Ozaki S, Kubota H, Ito H et al (1998) Intratumoral cytogenetic heterogeneity detected by comparative genomic hybridization and laser scanning cytometry in human gliomas. Cancer Res 58:4694-4700

33. Wemmert S, Romeike BF, Ketter R, Steudel WI, Zang KD et al (2006) Intratumoral genetic heterogeneity in pilocytic astrocytomas revealed by CGH-analysis of microdissected tumor cells and FISH on tumor tissue sections. Int J Oncol 28:353-360
34. Lopez-Gines C, Cerda-Nicolas M, Gil-Benso R, Pellin A, LopezGuerrero JA et al (2005) Association of chromosome 7, chromosome 10 and EGFR gene amplification in glioblastoma multiforme. Clin Neuropathol 24:209-218

35. Necesalova E, Vranova V, Kuglik P, Cejpek P, Jarosova M et al (2007) Incidence of the main genetic markers in glioblastoma multiforme is independent of tumor topology. Neoplasma 54: 212-218

36. Shinojima N, Tada K, Shiraishi S, Kamiryo T, Kochi M et al (2003) Prognostic value of epidermal growth factor receptor in patients with glioblastoma multiforme. Cancer Res 63:6962-6970

37. McLendon RE, Turner K, Perkinson K, Rich J (2007) Second messenger systems in human gliomas. Arch Pathol Lab Med 131:1585-1590

38. Scaltriti M, Baselga J (2006) The epidermal growth factor receptor pathway: a model for targeted therapy. Clin Cancer Res $12: 5268-5272$

39. Li L, Ross AH (2007) Why is PTEN an important tumor suppressor? J Cell Biochem 102:1368-1374

40. Hlobilkova A, Ehrmann J, Sedlakova E, Krejci V, Knizetova P et al (2007) Could changes in the regulation of the PI3K/PKB/Akt signaling pathway and cell cycle be involved in astrocytic tumor pathogenesis and progression? Neoplasma 54:334-341

41. Rao RD, James CD (2004) Altered molecular pathways in gliomas: an overview of clinically relevant issues. Semin Oncol 31:595-604

42. Ohtani N, Yamakoshi K, Takahashi A, Hara E (2004) The p16INK4a-RB pathway: molecular link between cellular senescence and tumor suppression. J Med Invest 51:146-153

43. Jones DT, Kocialkowski S, Liu L, Pearson DM, Backlund LM et al (2008) Tandem duplication producing a novel oncogenic BRAF fusion gene defines the majority of pilocytic astrocytomas. Cancer Res 68:8673-8677

44. Bar EE, Lin A, Tihan T, Burger PC, Eberhart CG (2008) Frequent gains at chromosome $7 \mathrm{q} 34$ involving BRAF in pilocytic astrocytoma. J Neuropathol Exp Neurol 67:878-887

45. Jendrossek V, Kugler W, Erdlenbruch B, Eibl H, Lakomek M (2001) Induction of differentiation and tetraploidy by long-term treatment of C6 rat glioma cells with erucylphosphocholine. Int J Oncol 19:673-680

46. Ganem NJ, Storchova Z, Pellman D (2007) Tetraploidy, aneuploidy and cancer. Curr Opin Genet Dev 17:157-162

47. Liu Y, Yeh N, Zhu XH, Leversha M, Cordon-Cardo C et al (2007) Somatic cell type specific gene transfer reveals a tumor-promoting function for p21(Waf1/Cip1). EMBO J 26:4683-4693

48. Tews B, Felsberg J, Hartmann C, Kunitz A, Hahn M et al (2006) Identification of novel oligodendroglioma-associated candidate tumor suppressor genes in $1 \mathrm{p} 36$ and $19 \mathrm{q} 13$ using microarraybased expression profiling. Int J Cancer 119:792-800

49. Barbashina V, Salazar P, Holland EC, Rosenblum MK, Ladanyi M (2005) Allelic losses at 1p36 and 19q13 in gliomas: correlation with histologic classification, definition of a $150-\mathrm{kb}$ minimal deleted region on 1p36, and evaluation of CAMTA1 as a candidate tumor suppressor gene. Clin Cancer Res 11:1119-1128

50. Weller M, Berger H, Hartmann C, Schramm J, Westphal M et al (2007) Combined 1p/19q loss in oligodendroglial tumors: predictive or prognostic biomarker? Clin Cancer Res 13:6933-6937

51. Jenkins RB, Blair H, Ballman KV, Giannini C, Arusell RM et al (2006) A t $(1 ; 19)(\mathrm{q} 10 ; \mathrm{p} 10)$ mediates the combined deletions of $1 \mathrm{p}$ and $19 \mathrm{q}$ and predicts a better prognosis of patients with oligodendroglioma. Cancer Res 66:9852-9861

52. Felsberg J, Erkwoh A, Sabel MC, Kirsch L, Fimmers R et al (2004) Oligodendroglial tumors: refinement of candidate regions on chromosome arm $1 \mathrm{p}$ and correlation of $1 \mathrm{p} / 19 \mathrm{q}$ status with survival. Brain Pathol 14:121-130 
53. Jenkins RB, Curran W, Scott CB, Cairncross G (2001) Pilot evaluation of $1 p$ and $19 q$ deletions in anaplastic oligodendrogliomas collected by a national cooperative cancer treatment group. Am J Clin Oncol 24:506-508

54. Ohgaki H, Kleihues P (2005) Population-based studies on incidence, survival rates, and genetic alterations in astrocytic and oligodendroglial gliomas. J Neuropathol Exp Neurol 64:479-489

55. Qu M, Olofsson T, Sigurdardottir S, You C, Kalimo H et al (2007) Genetically distinct astrocytic and oligodendroglial components in oligoastrocytomas. Acta Neuropathol 113:129-136

56. Reddy KS (2008) Assessment of 1p/19q deletions by fluorescence in situ hybridization in gliomas. Cancer Genet Cytogenet 184:77-86
57. Jones DT, Ichimura K, Liu L, Pearson DM, Plant K et al (2006) Genomic analysis of pilocytic astrocytomas at $0.97 \mathrm{Mb}$ resolution shows an increasing tendency toward chromosomal copy number change with age. J Neuropathol Exp Neurol 65:10491058

58. Lima-Ramos V, Pacheco-Figueiredo L, Costa S, Pardal F, Silva A et al (2008) TP53 codon 72 polymorphism in susceptibility, overall survival, and adjuvant therapy response of gliomas. Cancer Genet Cytogenet 180:14-19

59. Chang Y, Berenson JR, Wang Z, Deuel TF (2006) Dominant negative pleiotrophin induces tetraploidy and aneuploidy in U87MG human glioblastoma cells. Biochem Biophys Res Commun 351:336-339 